\title{
Anti-Synchronization of Fractional-Order Chaotic Circuit with Memristor via Periodic Intermittent Control
}

\author{
Fanqi Meng $\left(\mathbb{D},{ }^{1,2}\right.$ Xiaoqin Zeng, ${ }^{2}$ Zuolei Wang $\mathbb{D},{ }^{1}$ and Xinjun Wang ${ }^{2}$ \\ ${ }^{1}$ School of Mathematics and Statistics, Yancheng Teachers University, Yancheng, China \\ ${ }^{2}$ Computer and Information Engineering College, Hohai University, Nanjing, China \\ Correspondence should be addressed to Fanqi Meng; fqmeng@hhu.edu.cn and Zuolei Wang; wangzuolei1971@163.com
}

Received 3 June 2019; Accepted 19 November 2019; Published 22 January 2020

Academic Editor: Carlo Bianca

Copyright (C) 2020 Fanqi Meng et al. This is an open access article distributed under the Creative Commons Attribution License, which permits unrestricted use, distribution, and reproduction in any medium, provided the original work is properly cited.

\begin{abstract}
In this paper, the anti-synchronization of fractional-order chaotic circuit with memristor (FCCM) is investigated via a periodic intermittent control scheme. Based on the principle of periodic intermittent control and the Lyapunov stability theory, a novel criterion is adopted to realize the anti-synchronization of FCCM. Finally, some examples of numerical simulations are exploited to verify the feasibility of theoretical analysis.
\end{abstract}

\section{Introduction}

Fractional calculus has a history of more than 300 years. It is worth pointing out that fractional-order system has provided infinite memory and more accurately describes natural phenomena than other integer order systems [1]. In recent decades, fractional differential equations due to its potential applications in many fields such as fluid mechanics [2], physics [3], encryption [4], and control processing [5]. Especially, the dynamical behavior of fractional order systems exhibits chaos, such as fractional-order Chen system, fractional-order Chua system, fractional-order Lorenz system, and fractional-order Lü system, etc., [6].

The memristor was firstly raised by Chua [7], but it had not aroused any attention until 2008 when the invention of the memristor had been published by the researchers in Hewlett-Packard lab $[8,9]$. The memristor could "remember" its state when the voltage is turned off. Because of the characteristics of memristor, the potential applications of the chaotic system with memristor have been discovered in quite a few fields such as cryptography, filter, image encryption, etc., [10-15]. Therefore, the behaviors and properties of memristor have attracted the attention of many researchers attention.

Meanwhile, many scholars investigate the synchronization problems [16-18], especially the synchronization of fractional-order chaotic systems such as lag-synchronization [19], projective synchronization [20], impulsive synchronization
[21], and anti-synchronization [22]. Examples of synchronization occur in different fields of engineering and science like coupled cardiac, circuits in electronics and respiratory systems in physiology, and coupled laser systems in nonlinear optics. Many synchronization methods have been put forward for chaotic systems, such as sliding mode control method [23, 24], impulsive control method [25,26], active control method [27], periodic intermittent control method [28, 29], etc.

Intermittent control, which was first introduced to control linear econometric models in [30], has been widely used in engineering fields such as manufacturing, transportation, and communication for its practical and easy implementation in engineering control. Intermittent control is a discontinuous control method, its control input is activated during certain nonzero time intervals and closed during other time intervals [31]. Therefore, compared with the continuous control methods, intermittent control is more economical and efficient [32]. Recently, much effort has been devoted to study the issue of stabilization and synchronization of chaotic systems and dynamical networks by using intermittent control, and many important and interesting results have been obtained [33-38]. In [33], pinning synchronization for directed networks with node balance via adaptive intermittent control was researched. Zhang et al. [34] studied the lag synchronization for fractional-order memristive neural networks via periodic intermittent control. References $[35,36]$ proposed the finite-time synchronization via periodic intermittent control. Liu et al. 
[37] considered the cluster synchronization for delayed complex networks via periodically intermittent pinning control. The exponential synchronization of complex delayed dynamical networks via pinning periodically intermittent control was investigated in reference [38]. To the best of our knowledge, there is no study on the anti-synchronization of FCCM via a periodic intermittent control method. In real applications, the anti-synchronization of FCCM has great value in many areas, such as image encryption, cryptography, and chaotic radar.

Motivated by the above discussions, we propose a periodic intermittent control method for the anti-synchronization of FCCM in this paper. Based on the lyapunov stability theory, a novel and useful criterion of periodic intermittent control is developed by using the differential inequality method. Finally, we have illustrated the effectiveness and feasibility of the proposed approaches by numerical simulations.

This paper is arranged as follows: Section 2 describes some fundamental definitions, the lemmas and the model formulation. The anti-synchronization of FCCM via periodic intermittent control is discussed in Section 3. In Section 4, some numerical examples are provided to illustrate the effectiveness of the theoretical approach. The conclusions are put forward in the last section.

\section{Preliminaries}

In this paper, let $R^{n}$ denote the $\mathrm{n}$-dimensional Euclidean space, $x=\left(x_{1}, x_{2}, x_{3}, x_{4}\right)^{T} \in R^{4}, y=\left(y_{1}, y_{2}, y_{3}, y_{4}\right)^{T} \in R^{4}$. In this section, some fundamental definitions and lemmas are recalled. In addition, we introduce fractional-order generalization form of the chaotic circuit with memristor.

Definition 1 [39]. The caputo's fractional derivative for a function $z(t):[0,+\infty) \rightarrow R$ is defined by

$$
{ }_{0}^{c} D_{t}^{\alpha} z(t)=\frac{1}{\Gamma(n-\alpha)} \int_{0}^{t} \frac{z^{n}(\tau)}{(t-\tau)^{\alpha-n+1}} d \tau,
$$

where $t \geq 0, \alpha$ is fractional-order, $n \in Z^{+}, n-1<\alpha<n$, and $\Gamma(\cdot)$ is the gamma function.

Particularly, when $0<\alpha<1$,

$$
{ }_{0}^{c} D_{t}^{\alpha} z(t)=\frac{1}{\Gamma(1-\alpha)} \int_{0}^{t} \frac{z^{\prime}(\tau)}{(t-\tau)^{\alpha}} d \tau .
$$

Definition 2 [39]. The Mittag-Leffler function of $\alpha$ is defined as

$$
E_{\alpha}(x)=\sum_{m=0}^{\infty} \frac{x^{m}}{\Gamma(m \alpha+1)},
$$

where $\alpha>0$ and $x \in C$.

Lemma 1 [40]. Suppose $z(t) \in R^{n}$ is a differentiable function and continuous. For $\alpha \in(0,1)$, the following inequality holds

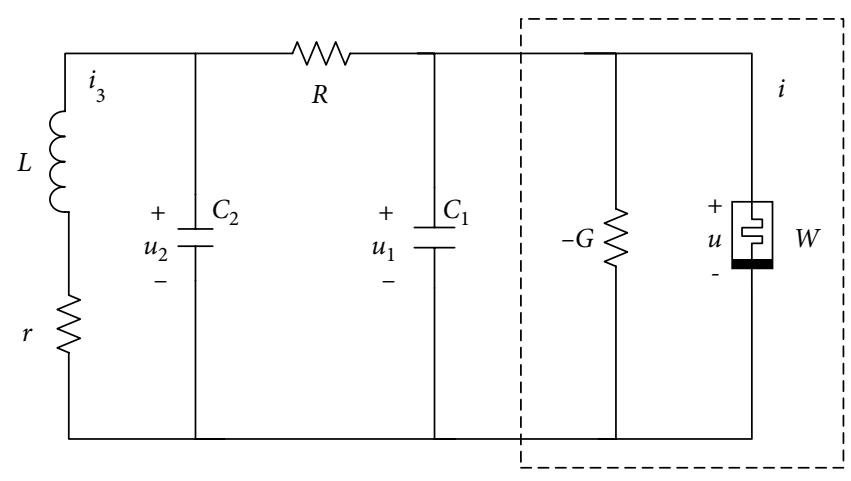

Memristor

FIgURE 1: The chaotic circuit with memristor.

$$
\frac{1}{2}{ }_{0}^{c} D_{t}^{\alpha} z^{T}(t) z(t) \leq z^{T}(t)_{0}^{c} D_{t}^{\alpha} z(t) .
$$

Lemma 2 [41]. For $\alpha \in(0,1), x \in R$, and $x>0, E_{\alpha}(x)$ is a monotone increasing function.

Lemma 3 [39]. Let $V(t)$ be a continuous function on $\left[t_{0},+\infty\right)$ and satisfies

$$
{ }_{t_{0}}^{c} D_{t}^{\alpha} V(t) \leq \theta V(t)
$$

where $0<\alpha<1$ and $\theta$ is a constant, then

$$
V(t) \leq V\left(t_{0}\right) E_{\alpha}\left(\theta\left(t-t_{0}\right)^{\alpha}\right)
$$

Lemma 4 [42]. Let $0<\alpha<1, \lambda>0$, and $t \geq 0$, the following inequality holds

$$
0 \leq E_{\alpha}\left(-\lambda t^{\alpha}\right) \leq 1
$$

Moreover, $E_{\alpha}(0)=1$.

According to the chaotic circuit with memristor [43] as shown in Figure 1, the flux-controlled memristor is defined by

$$
\begin{aligned}
q(\phi) & =a \phi+b \phi^{3}, \\
W(\phi) & =\frac{d q(\phi)}{d \phi}=a+3 b \phi^{2},
\end{aligned}
$$

where $\phi$ is the flux, $W(\phi)$ is the memductance, and $a$ and $b$ are constants.

Similar to [43], let $x_{1}=u_{1}, x_{2}=u_{2}, x_{3}=i_{3}, x_{4}=\phi, \beta=1 / C_{1}$, $\psi=1 / L_{1}, \xi=G, \gamma=r / L, R=1$, and $C_{2}=1$, then the mathematical model of the chaotic circuit with memristor as follows

$$
\begin{aligned}
& \dot{x}_{1}=\beta\left(\xi x_{1}-x_{1}+x_{2}-W\left(x_{4}\right) x_{1}\right), \\
& \dot{x}_{2}=x_{1}-x_{2}+x_{3}, \\
& \dot{x}_{3}=-\psi x_{2}-\gamma x_{3}, \\
& \dot{x}_{4}=x_{1} .
\end{aligned}
$$

Refer to the above model, the fractional-order generalization according to (9) is described as 


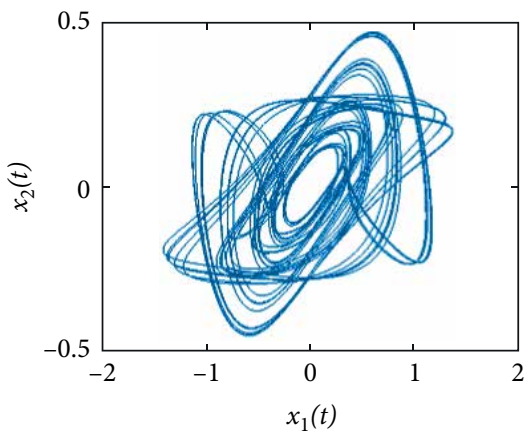

(a)

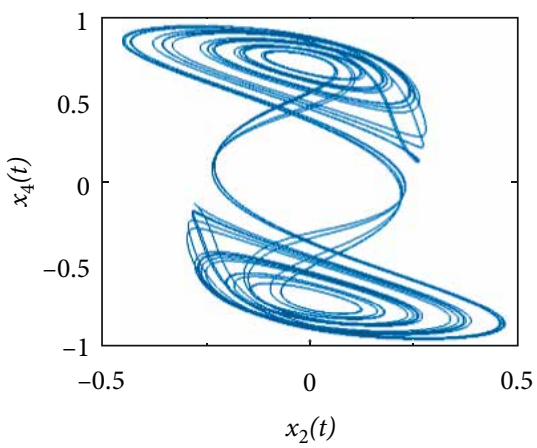

(c)

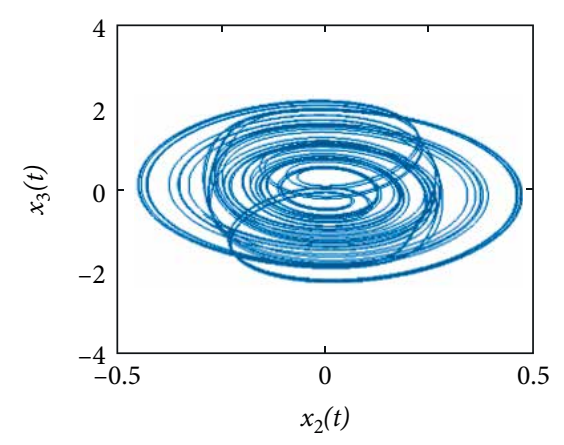

(b)

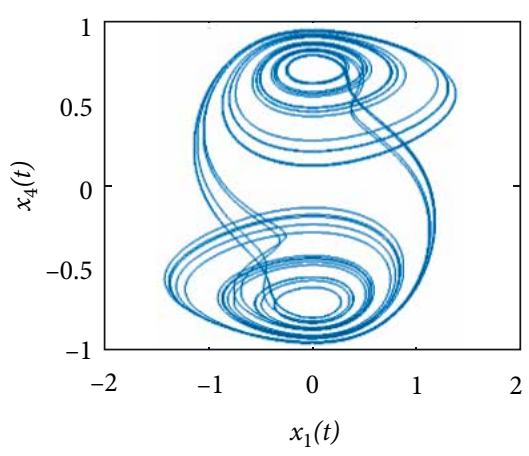

(d)

Figure 2: The chaotic attractor of FCCM, (a) $x_{1}(t), x_{2}(t)$; (b) $x_{2}(t), x_{3}(t)$; (c) $x_{2}(t), x_{4}(t)$; (d) $x_{1}(t), x_{4}(t)$.

$$
\begin{aligned}
& { }_{0}^{c} D_{t}^{\alpha} x_{1}=\beta\left(\xi x_{1}-x_{1}+x_{2}-W\left(x_{4}\right) x_{1}\right), \\
& { }_{0}^{c} D_{t}^{\alpha} x_{2}=x_{1}-x_{2}+x_{3}, \\
& { }_{0}^{c} D_{t}^{\alpha} x_{3}=-\psi x_{2}-\gamma x_{3}, \\
& { }_{0}^{c} D_{t}^{\alpha} x_{4}=x_{1} .
\end{aligned}
$$

Usually, in order to obtain the chaotic phenomena, the parameters are selected as $\alpha=0.985, \beta=10, \psi=100 / 7, \xi=9 / 7$, $\gamma=0.1, a=1 / 7, b=2 / 7$, and the initial value $(0,0.01,0,0)^{T}$ to system (10). The simulation results are shown in Figure 2. by

Let $x=\left[x_{1}, x_{2}, x_{3}, x_{4}\right]^{T}$, the system (10) can be described

$$
{ }_{0}^{c} D_{t}^{\alpha} x=A x+\phi(x)
$$

where

$$
A=\left[\begin{array}{cccc}
\beta(\xi-1) & \beta & 0 & 0 \\
1 & -1 & 1 & 0 \\
0 & -\psi & -\gamma & 0 \\
1 & 0 & 0 & 0
\end{array}\right], \quad \phi(x)=\left[\begin{array}{c}
-\beta W\left(x_{4}\right) x_{1} \\
0 \\
0 \\
0
\end{array}\right]
$$

and $a, b, \gamma, \xi, \beta, \psi$ are positive constants.

To investigate the anti-synchronization of FCCM, the drive system can be rewritten as

$$
{ }_{0}^{c} D_{t}^{\alpha} x(t)=A x(t)+\phi(x(t)) .
$$

Similarly, the response system with the controller can be described as

$$
{ }_{0}^{c} D_{t}^{\alpha} y(t)=A y(t)+\phi(y(t))+u(t),
$$

where $u(t)$ is the intermittent periodical controller which is proposed by

$$
u(t)=\begin{array}{ll}
-k(x(t)+y(t)), & m T \leq t<m T+\sigma \\
0, & m T+\sigma \leq t<(m+1) T
\end{array}
$$

here $k>0$ is a positive constant, $T>0$ is the control period, $0<\sigma<T$ is called the control width, and $m=0,1,2, \cdots$.

Let $e(t)=y(t)+x(t)$ be the synchronization error between system (13) and system (14), the error system can be obtained by

$$
\begin{aligned}
& { }_{0}^{c} D_{t}^{\alpha} e(t)=A e(t)+\phi(e(t))-k e(t), \quad m T \leq t \leq m T+\sigma, \\
& { }_{0}^{c} D_{t}^{\alpha} e(t)=A e(t)+\phi(e(t)), \quad m T+\sigma<t<(m+1) T,
\end{aligned}
$$

where

$$
\begin{aligned}
\phi(e(t)) & =\phi(x(t))+\phi(y(t)) \\
& =\left[-\beta W\left(x_{4}(t)\right) x_{1}(t)-\beta W\left(y_{4}(t)\right) y_{1}(t), 0,0,0\right]^{T} .
\end{aligned}
$$

\section{Main Result}

In this section, the anti-synchronization problem of FCCM via periodic intermittent control is investigated. First of all, we propose the following assumption.

Assumption 1. It can be seen from Figure (2) that system (10) is a chaotic system with bounders, we assume $M_{1}$ and $M_{2}$ are positive constants, such that

$$
\left|x_{1}(t)\right| \leq M_{1}, \quad\left|x_{4}(t)\right| \leq M_{2}
$$




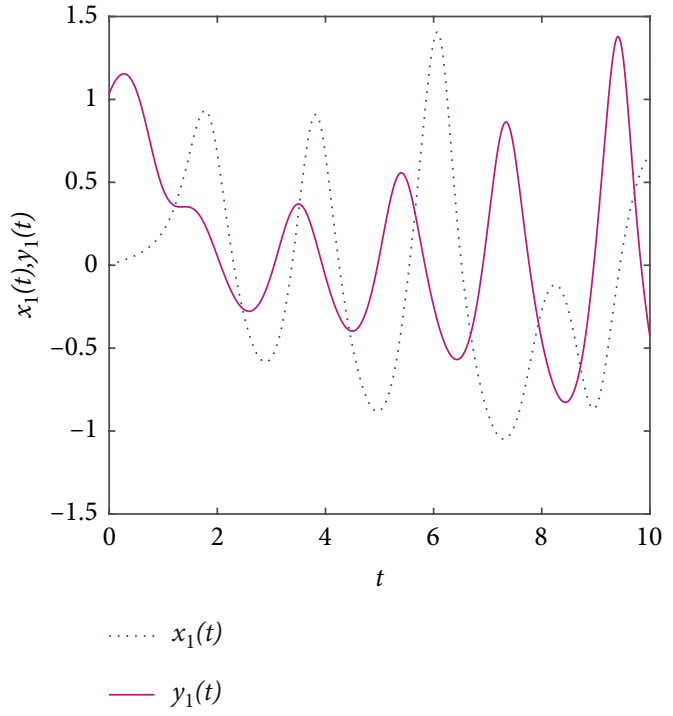

(a)

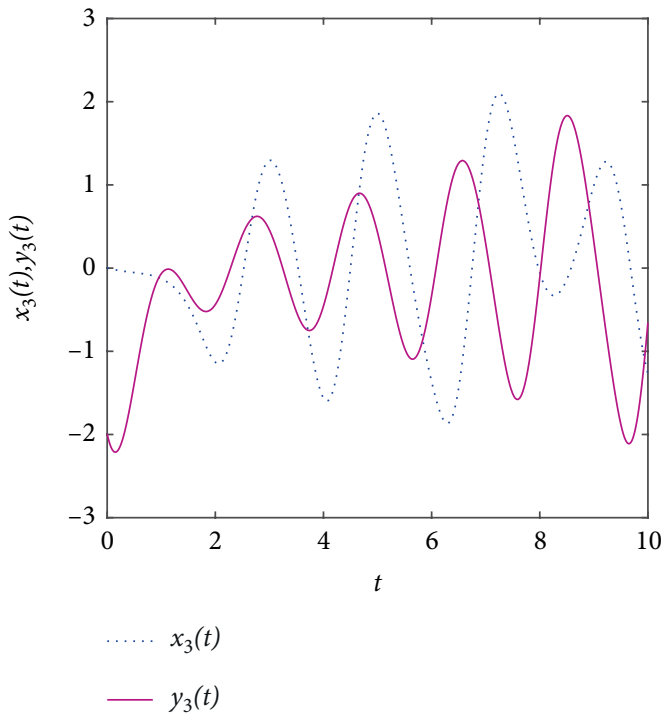

(c)

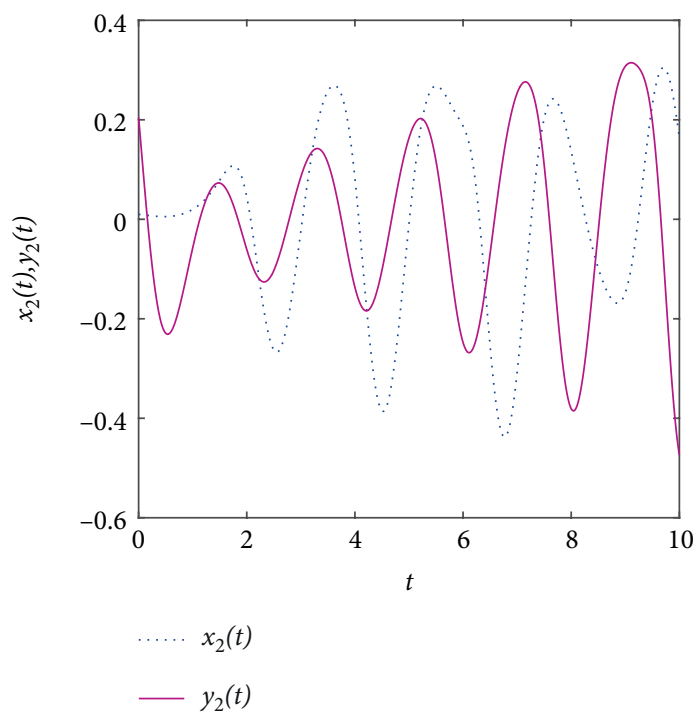

(b)

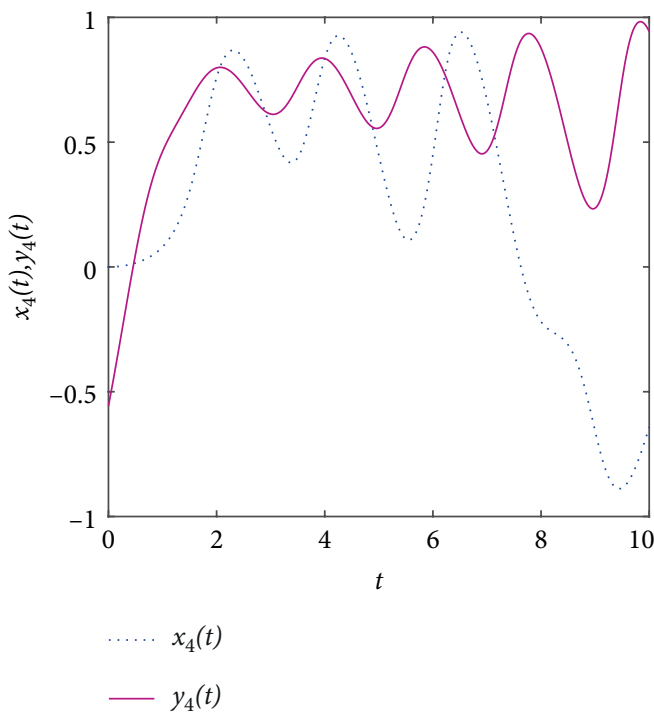

(d)

FIGURE 3: Dynamical behaviors of the drive system (13) and the response system (14) without periodic intermittent controller (15), where $\alpha=0.985$, (a) $x_{1}(t), y_{1}(t)$; (b) $x_{2}(t), y_{2}(t)$; (c) $x_{3}(t), y_{3}(t)$; (d) $x_{4}(t), y_{4}(t)$.

Then, we derive the anti-synchronization criteria for the FCCM according to periodic intermittent control scheme and Assumption 1 in the Theorem 1.

Theorem 1. Suppose Assumption 1 holds. The systems (13) and (14) can be anti-synchronized under the periodic intermittent controller (15) if the following conditions are satisfied:

$$
\begin{gathered}
2\left(\lambda_{A}+3 \beta b M_{1} M_{2}-k\right)+\eta_{1} \leq 0, \\
2\left(\lambda_{A}+3 \beta b M_{1} M_{2}\right)-\eta_{2} \leq 0,
\end{gathered}
$$

$$
E_{\alpha}\left(-\eta_{1} \sigma^{\alpha}\right) E_{\alpha}\left(\eta_{2}(T-\sigma)^{\alpha}\right)<1
$$

where $\lambda_{A}$ is the largest eigenvalue of $A, \eta_{1}$, and $\eta_{2}$ are nonnegative constants.

Proof. Construct the following Lyapunov function.

$$
V(t)=\frac{1}{2} e^{T}(t) e(t)
$$

Taking the time derivative of $V(t)$ along the solution $e(t)$ of the system (22). From Lemma 1, when $m T \leq t \leq m T+\sigma$, $m=0,1,2, \ldots$, we have 


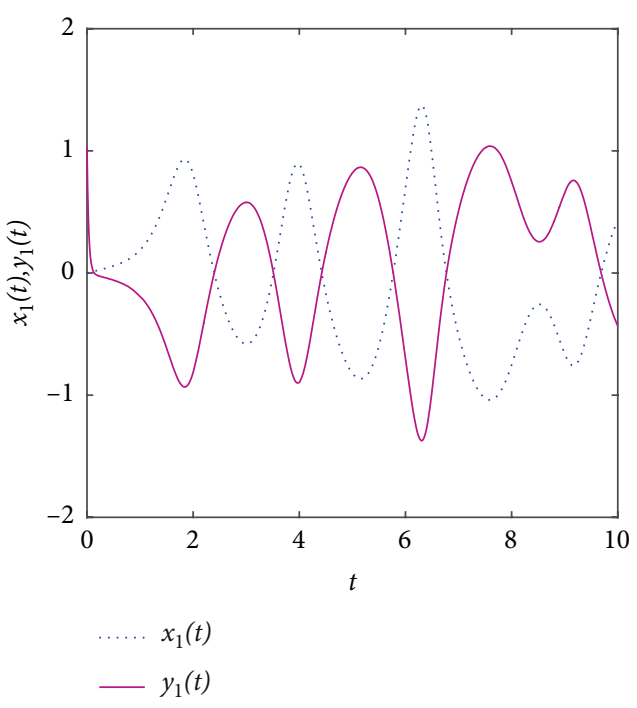

(a)

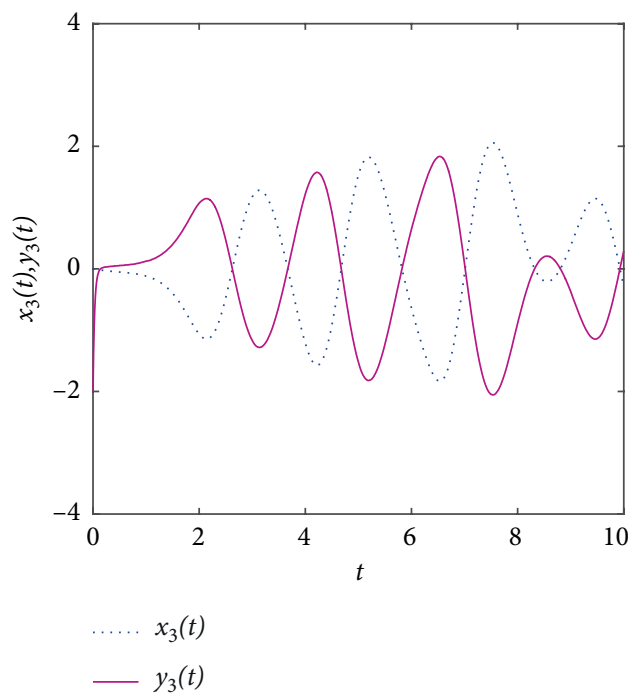

(c)

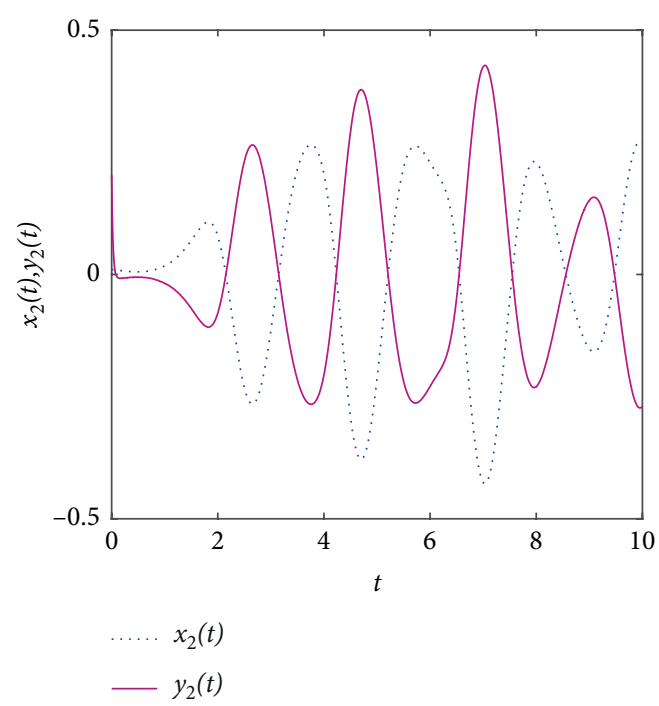

(b)

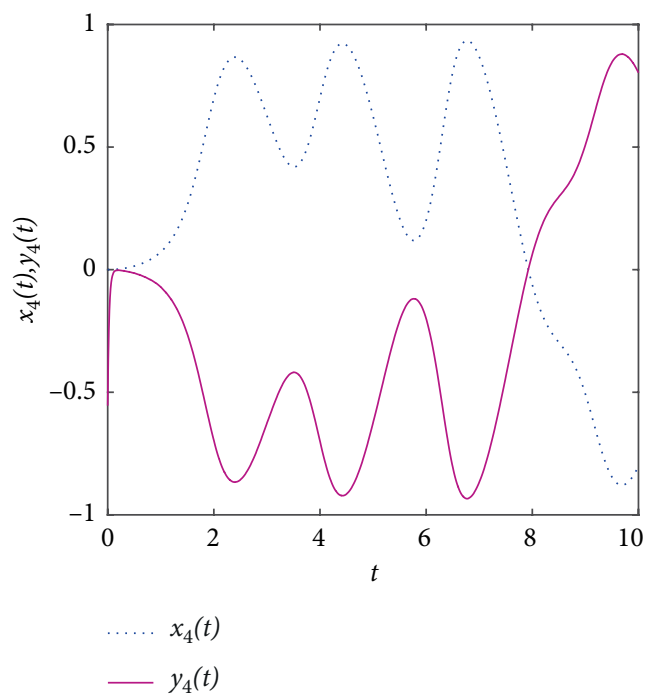

(d)

FIGURE 4: Dynamical behaviors of the drive system (13) and the response system (14) with periodic intermittent controller (15), where $\alpha=0.985, \eta_{1}=30, \eta_{2}=34, k=33, T=1$, and $\sigma=0.8$, (a) $x_{1}(t), y_{1}(t) ;(\mathrm{b}) x_{2}(t), y_{2}(t) ;(\mathrm{c}) x_{3}(t), y_{3}(t) ;(\mathrm{d}) x_{4}(t), y_{4}(t)$.

$$
\begin{aligned}
{ }_{m T}^{c} D_{t}^{\alpha} V(t) \leq & e^{T}(t)_{m T}^{c} D_{t}^{\alpha} e(t) \\
= & e^{T}(t)(A e(t)+\phi(e(t))-k e(t)) \\
= & e^{T}(t) A e(t)+e^{T}(t) \phi(e(t))-k e^{T}(t) e(t) \\
= & e^{T}(t) A e(t)-\beta\left[a e_{1}^{2}(t)+3 b\left(y_{4}^{2}(t) y_{1}(t)+y_{4}^{2}(t) x_{1}(t)\right.\right. \\
& \left.\left.x_{4}^{2}(t) x_{1}(t)-y_{4}^{2}(t) x_{1}(t)\right) e_{1}(t)\right]-k e^{T}(t) e(t) \\
\leq & \lambda_{A} e^{T}(t) e(t)+3 \beta b M_{1} M_{2} e^{T}(t) e(t)-k e^{T}(t) e(t) \\
= & \left(\lambda_{A}+3 \beta b M_{1} M_{2}-k\right) e^{T}(t) e(t) .
\end{aligned}
$$

According to the condition (19), we have

$$
{ }_{m T}^{c} D_{t}^{\alpha} V(t) \leq-\eta_{1} V(t) .
$$

By Lemma 3, when $m T \leq t \leq m T+\sigma$, we have

$$
V(t) \leq V(m T) E_{\alpha}\left(-\eta_{1}(t-m T)^{\alpha}\right) .
$$

Similarly, when $m T+\sigma<t<m T+T, m=0,1,2, \ldots$ we have

$$
\begin{aligned}
{ }_{m T+\sigma}^{c} D_{t}^{\alpha} V(t) & \leq e^{T}(t)_{m T+\sigma}^{c} D_{t}^{\alpha} e(t) \\
& =e^{T}(t)(A e(t)+\phi(e(t))) \\
& =\left(\lambda_{A}+3 \beta b M_{1} M_{2}\right) e^{T}(t) e(t) .
\end{aligned}
$$

According to condition (20), we have

$$
{ }_{m T+\sigma}^{c} D_{t}^{\alpha} V(t) \leq \eta_{2} V(t) .
$$

From Lemma 3, when $m T+\sigma<t<(m+1) T$, we have

$$
V(t) \leq V(m T+\sigma) E_{\alpha}\left(\eta_{2}(t-m T-\sigma)^{\alpha}\right) .
$$

From inequality (25) and (28), we summarize that: When $0 \leq t \leq \sigma$, we have

$$
V(t) \leq V(0) E_{\alpha}\left(-\eta_{1} t^{\alpha}\right) .
$$

When $\sigma<t<T$, we obtain 


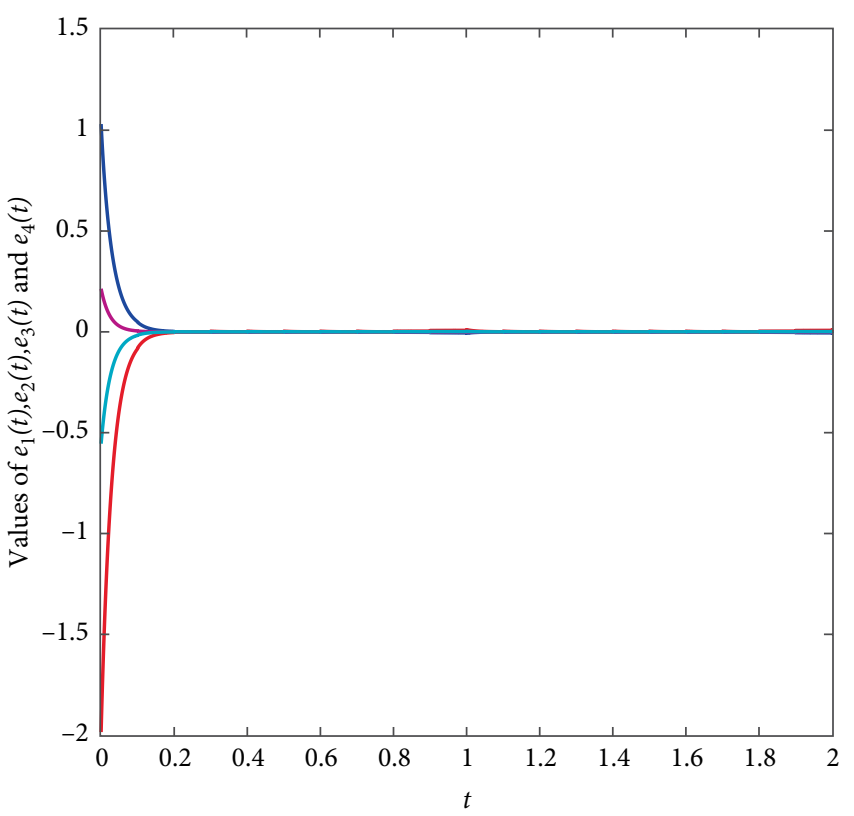

FIGURE 5: Synchronization errors between systems (13) and (14) with periodic intermittent controller (15), where $\alpha=0.985$, $\eta_{1}=30, \eta_{2}=34, k=33, T=1$, and $\sigma=0.8$.

$$
\begin{aligned}
V(t) & \leq V(\sigma) E_{\alpha}\left(\eta_{2}(t-\sigma)^{\alpha}\right) \\
& \leq V(0) E_{\alpha}\left(-\eta_{1} \sigma^{\alpha}\right) E_{\alpha}\left(\eta_{2}(t-\sigma)^{\alpha}\right) .
\end{aligned}
$$

When $T \leq t \leq T+\sigma$, we have

$$
\begin{aligned}
V(t) & \leq V(T) E_{\alpha}\left(-\eta_{1}(t-T)^{\alpha}\right) \\
& \leq V(0) E_{\alpha}\left(-\eta_{1} \sigma^{\alpha}\right) E_{\alpha}\left(\eta_{2}(T-\sigma)^{\alpha}\right) E_{\alpha}\left(-\eta_{1}(t-T)^{\alpha}\right) .
\end{aligned}
$$

When $T+\sigma<t<2 T$, we obtain

$$
\begin{aligned}
V(t) \leq & V(T+\sigma) E_{\alpha}\left(\eta_{2}(t-T-\sigma)^{\alpha}\right) \\
\leq & V(0) E_{\alpha}\left(-\eta_{1} \sigma^{\alpha}\right) E_{\alpha}\left(\eta_{2}(T-\sigma)^{\alpha}\right) E_{\alpha}\left(-\eta_{1}(T+\sigma-T)^{\alpha}\right) \\
& E_{\alpha}\left(\eta_{2}(t-T-\sigma)^{\alpha}\right) \\
\leq & V(0) E_{\alpha}\left(-\eta_{1} \sigma^{\alpha}\right)^{2} E_{\alpha}\left(\eta_{2}(T-\sigma)^{\alpha}\right) E_{\alpha}\left(\eta_{2}(t-T-\sigma)^{\alpha}\right) .
\end{aligned}
$$

By induction, when $m T \leq t \leq m T+\sigma$, we have

$$
\begin{aligned}
V(t) & \leq V(m T) E_{\alpha}\left(-\eta_{1}(t-m T)^{\alpha}\right) \\
& \leq V(0) E_{\alpha}\left(-\eta_{1} \sigma^{\alpha}\right)^{m} E_{\alpha}\left(\eta_{2}(T-\sigma)^{\alpha}\right)^{m} E_{\alpha}\left(-\eta_{1}(t-m T)^{\alpha}\right) .
\end{aligned}
$$

From Lemma 4, we obtain

$$
V(t) \leq V(0)\left(E_{\alpha}\left(-\eta_{1} \sigma^{\alpha}\right) E_{\alpha}\left(\eta_{2}(T-\sigma)^{\alpha}\right)\right)^{m} .
$$

when $m T+\sigma<t<(m+1) T$, we have

$$
\begin{aligned}
V(t) \leq & V(m T+\sigma) E_{\alpha}\left(\eta_{2}(t-m T-\sigma)^{\alpha}\right) \\
\leq & V(0) E_{\alpha}\left(-\eta_{1} \sigma^{\alpha}\right)^{m} E_{\alpha}\left(\eta_{2}(T-\sigma)^{\alpha}\right)^{m} \\
& E_{\alpha}\left(-\eta_{1} \sigma^{\alpha}\right) E_{\alpha}\left(\eta_{2}(t-m T-\sigma)^{\alpha}\right) .
\end{aligned}
$$

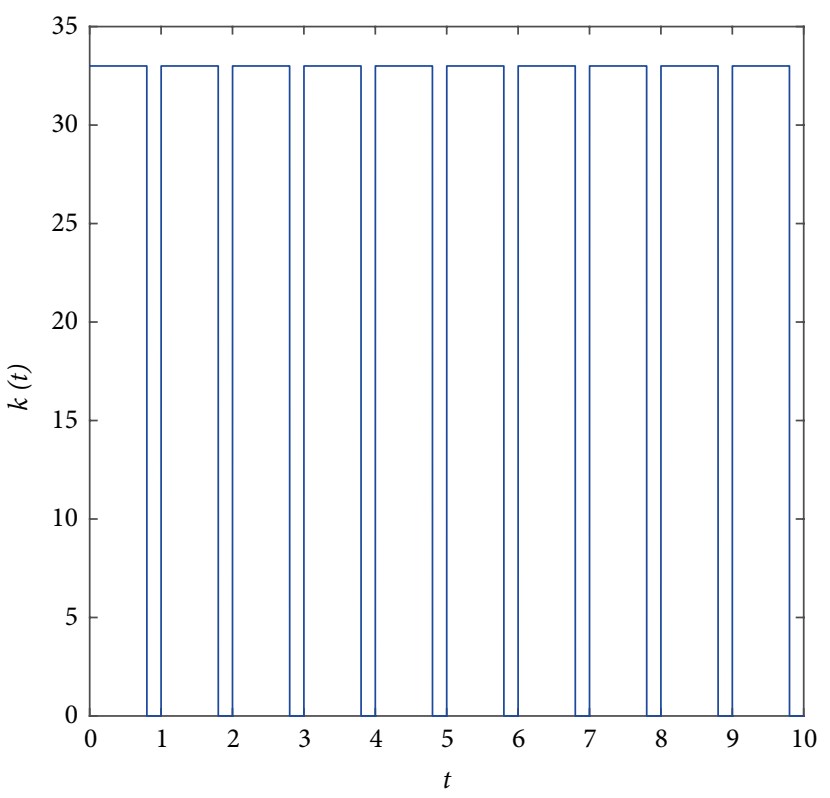

FIgURE 6: Time evolution of intermittent feedback control gain $k(t)$ for error system (16) with periodic intermittent controller (15), where $T=1, \sigma=0.8$.

Therefore, from inequality (34) and (35), we have

$$
\begin{gathered}
V(0)\left(E_{\alpha}\left(-\eta_{1} \sigma^{\alpha}\right) E_{\alpha}\left(\eta_{2}(T-\sigma)^{\alpha}\right)\right)^{m}, \\
m T \leq t \leq m T+\sigma \\
V(t) \leq \quad V(0)\left(E_{\alpha}\left(-\eta_{1} \sigma^{\alpha}\right) E_{\alpha}\left(\eta_{2}(T-\sigma)^{\alpha}\right)\right)^{m} E_{\alpha}\left(\eta_{2}(t-m T-\sigma)^{\alpha}\right), \\
m T+\sigma<t<(m+1) T .
\end{gathered}
$$

According to condition (21), since $m \rightarrow \infty$ when $t \rightarrow \infty$, we can obtain that

$$
V(t) \rightarrow 0
$$

Hence, $\|e(t)\| \rightarrow 0$ as $t \rightarrow \infty$. It follows that the error system (16) is global stable. It means that system (14) is antisynchronized with system (13). This completes the proof.

Remark 1. Because $T, \sigma, \eta_{1}$, and $\eta_{2}$ are nonnegative constants, we can get $0 \leq E_{\alpha}\left(-\eta_{1} \sigma^{\alpha}\right) \leq 1, E_{\alpha}(0)=1$ based on Lemma 4 . According to Lemma 2, when $T>\sigma, E_{\alpha}\left(\eta_{2}(T-\sigma)^{\alpha}\right) \geq 1$ is a monotone increasing function. Therefore, there are suitable constants $T, \sigma, \eta_{1}$, and $\eta_{2}$, which makes the condition (21) of Theorem 1 hold.

\section{Numerical Simulations}

In this section, some numerical simulations are given to illustrate the theoretical analysis.

Based on Figure 2 and Assumption 1, when $M_{1}=1.5$, $M_{2}=1$ are selected, $\lambda_{A}+3 \beta b M_{1} M_{2}=16.892$ is obtained. According to condition (20) of Theorem 1, we can obtain $\eta_{2} \geq 33.784$. Moreover we set $\alpha=0.985, \eta_{2}=34, T=1$, and $\sigma=0.8$, by condition (21) of Theorem 1 , we get 


$$
\begin{aligned}
& E_{\alpha}\left(-\eta_{1} \sigma^{\alpha}\right) E_{\alpha}\left(\eta_{2}(T-\sigma)^{\alpha}\right) \\
= & E_{0.985}\left(-\eta_{1} \times 0.8^{0.985}\right) E_{0.985}\left(34 \times(1-0.8)^{0.985}\right)<1,
\end{aligned}
$$

by Matlab calculation program, we can get that $\eta_{1}>27.57$ satisfies condition (21) of Theorem 1 . When we set $\eta_{1}=30$ and $k \geq 31.892$, the condition (19) of Theorem 1 holds.

Therefore, when we choose $\alpha=0.985, \eta_{1}=30, \eta_{2}=34$, $k=33, T=1$, and $\sigma=0.8$, all the conditions in Theorem 1 hold. The initial values of system (14) are the state variables of system (10) which freely-running within 2000 seconds. The initial values of the systems of (13) and (14) are set to be $(0$, $0.01,0,0)$ and $(1.0343,0.2048,-1.9919,-0.5568)$, respectively. It follows from Theorem 1 that the system (14) is anti-synchronized with system (13) under the periodic intermittent control.

Figure 3 shows the time evolution curves of systems (13) and (14) without periodic intermittent controller (15), indicating that there are different trajectories over time. By several conditions, Figure 4 displays the state trajectories of systems (13) and (14) with the periodic intermittent controller (15). Figure 5 depicts the error dynamics of the two systems with a periodic intermittent controller (15), which indicates that anti-synchronization can be implemented infinite time. The time evolution of the intermittent feedback control gain $k(t)$ is shown in Figure 6.

\section{Conclusions}

In this paper, the anti-synchronization of FCCM via periodic intermittent control has been achieved in finite time based on periodic intermittent control principle and Lyapunov stability theory. In addition, some numerical simulations have been provided to demonstrate the effectiveness of the proposed approach. The result will have potential applications for image encryption, cryptography, and chaotic radar. Our future research is to investigate the anti-synchronization of FCCM with time delay via nonperiodic intermittent control.

\section{Data Availability}

All data used to support the findings of this study are included within the article.

\section{Conflicts of Interest}

The authors declare that they have no conflicts of interests.

\section{Acknowledgments}

The authors are very grateful to the anonymous reviewers for their valuable comments. This work is supported by the National Science Foundation of China (Grants no. 51777180, 11771376, 11872327).

\section{References}

[1] K. B. Oldham and J. Spanier, "Introduction" in the Fractional Calculus: Theory and Applications of Differentiation and Integration to Arbitrary Order, Academic, NY, USA, pp. 1-16, 1974.

[2] D. Tripathil, S. Pandey, and S. Das, "Peristaltic flow of viscoelastic fluid with fractional Maxwell model through a channel," in Applied Mathematics and Computation, vol. 215, no. 10, pp. 3645-3654, 2010.

[3] R. Hilfer, in "Applications to problems in polymer physics and rheology," Applications of Fractional Calculus in Physics, World Scientific, New Jerseypp. 331-376, 2001.

[4] S. Vashisth, H. Singh, A. K. Yadav, and K. Singh, "Image encryption using fractional Mellin transform, structured phase filters, and phase retrieval," Optik, vol. 125, no. 18, pp. 5309-5315, 2014.

[5] C. A. Monje, Y. Q. Chen, B. M. Vinagre, D. Y. Xue, and V. FeliuBatlle, "Fundamentals of fractional-order control," FractionalOrder Systems and Controls, Fundamentals and Applications, Springer, London, pp. 75-84, 2010.

[6] I. Petráš, "Fractional-order chaotic systems," in Fractional-Order Nonlinearsystems: Modeling, Analysis and Simulation, Berlin Heidelberg, Springer, pp. 103-184, 2011.

[7] L. O. Chua, "Memristor-the missing circuit element," IEEE Transactions on Circuit Theory, vol. 18, no. 5, pp. 507-519, 1971.

[8] D. B. Strukov, G. S. Snider, D. R. Stewart, and R. S. Williams, "The missing memristor found," Nature, vol. 453, no. 7191, pp. $80-84,2009$.

[9] J. M. Tour and T. He, "Electronics: the fourth element," Nature, vol. 453, no. 7191, pp. 42-43, 2008.

[10] K. Eshraghian, K. R. Cho, O. Kavehei, S. K. Kang, D. Abbott, and S. M. S. Kang, "Memristor MOS content addressable memory (MCAM): hybrid architecture for future high performance search engines," IEEE Transaction on Very Large Scale Integration, vol. 19, pp. 1407-1417, 2010.

[11] X. L. Shi, S. K. Duan, L. D. Wang, T. W. Huang, and C. D. Li, "A novel memristive electronic synapse-based hermite chaotic neural network with application in cryptography," Neurocomputing, vol. 166, pp. 487-495, 2015.

[12] Y. Zhao, X. Zhang, J. Xu, and Y. Guo, "Identification of chaotic memristor systems based on piecewise adaptive legendre filters," Chaos, Solitons \& Fractals, vol. 81, pp. 315-319, 2015.

[13] J. W. Sun, X. T. Zhao, J. Fang, and Y. F. Wang, "Autonomous memristor chaotic systems of infinite chaotic attractors and circuitry realization," Nonlinear Dynamics, vol. 94, no. 4, pp. 1-9, 2018.

[14] J. Sun, G. Han, Y. Wang, H. Zhang, and L. Wu, "Hybrid memristor chaotic system," Journal of Nanoelectronics and Optoelectronics, vol. 13, no. 6, pp. 812-818, 2018.

[15] B. Bao, T. Jiang, G. Wang, P. Jin, H. Bao, and M. Chen, “Twomemristor-based Chua's hyperchaotic circuit with plane equilibrium and its extreme multistability," Nonlinear Dynamics, vol. 89, pp. 1157-1171, 2017.

[16] S. Zheng, "Intermittent impulsive projective synchronization in time-varying delayed dynamical network with variable structures," Complexity, vol. 21, no. S1, pp. 547-556, 2016.

[17] J. K. Zhao, Y. Wu, and Y. Y. Wang, "Generalized finite-time synchronization between coupled chaotic systems of different 
orders with unknown parameters," Nonlinear Dynamics, vol. 74, no. 3, pp. 479-485, 2013.

[18] G. L. Cai, S. Q. Jiang, S. M. Cai, and L. X. Tian, "Finite-time analysis of global projective synchronization on coloured networks," Pramana Journal of Physics, vol. 86, no. 3, pp. 545-554, 2015.

[19] Z. W. Sun, "Lag projective synchronization of two chaotic systems with different fractional orders," Journal of the Korean Physical Society, vol. 66, no. 8, pp. 1192-1199, 2015.

[20] Z. X. Ding and Y. Shen, "Projective synchronization of nonidentical fractional-order neural networks based on sliding mode controller," Neural Networks, vol. 76, pp. 97-105, 2016.

[21] W. Y. Ma, C. P. Li, and Y. J. Wu, "Impulsive synchronization of fractional Takagi-Sugeno fuzzy complex networks," Chaos, vol. 26, p. 084311, 2016.

[22] L. Zhang, C. L. Yu, and T. Liu, "Control of finite-time antisynchronization for variable-order fractional chaotic systems with unknown parameters," Nonlinear Dynamics, vol. 86, no. 3, pp. 1967-1980, 2016.

[23] J. W. Sun, Y. Wang, Y. F. Wang, and Y. Shen, "Finite-time synchronization between two complex-variable chaotic systems with unknown parameters via nonsingular terminal sliding mode control," Nonlinear Dynamics, vol. 85, pp. 1105-1117, 2016.

[24] J. W. Sun, Y. Y. Wu, G. Z. Cui, and Y. F. Wang, "Finite-time real combination synchronization of three complex-variable chaotic systems with unknown parameters via sliding mode control," Nonlinear Dynamics, vol. 88, no. 3, pp. 1677-1690, 2017.

[25] W. Xiong and J. Huang, "Finite-time control and synchronization for memristor-based chaotic system via impulsive adaptive strategy," Advances in Difference Equations, vol. 2016, no. 1, Article ID 101, 2016.

[26] X. Y. Wang, Y. L. Zhang, D. Lin, and N. Zhang, "Impulsive synchronisation of a class of fractional-order hyperchaotic systems," Chinese Physics B, vol. 20, p. 030506, 2011.

[27] S. K. Agrawal, M. Srivastava, and S. Das, "Synchronization of fractional order chaotic systems using active control method," Chaos, Solitons \& Fractals, vol. 45, no. 6, pp. 737-752, 2012.

[28] Y. Fan, H. Liu, Y. Zhu, and J. Mei, "Fast synchronization of complex dynamical networks with time-varying delay via periodically intermittent control," Neurocomputing, vol. 205, pp. 182-194, 2016.

[29] H.-L. Li, C. Hu, H. Jiang, Z. Teng, and Y.-L. Jiang, "Synchronization of fractional-order complex dynamical networks via periodically intermittent pinning control," Chaos, Solitons \& Fractals, vol. 103, pp. 357-363, 2017.

[30] C. Deissenberg, "Optimal control of linear econometric models with intermittent controls," Economics of Planning, vol. 16, no. 1, pp. 49-56, 1980.

[31] S. Wen, Z. Zeng, M. Z. Q. Chen, and T. Huang, "Synchronization of switched neural networks with communication delays via the event-triggered control," IEEE Transactions on Neural Networks and Learning Systems, vol. 28, no. 10, pp. 2334-2343, 2017.

[32] S. B. Ding and Z. S. Wang, "Lag quasi-synchronization for memristive neural networks with switching jumps mismatch," Neural Computing and Application, vol. 28, no. 12, pp. 4011-4022, 2016.

[33] C. Hu and H. Jiang, "Pinning synchronization for directed networks with node balance via adaptive intermittent control," Nonlinear Dynamics, vol. 80, no. 1-2, pp. 295-307, 2014.
[34] L. Z. Zhang, Y. Q. Yang, and F. Wang, "Lag synchronization for fractional-order memristive neural networks via periodic intermittent control," Nonlinear Dynamics, vol. 89, no. 3, pp. 367-381, 2017.

[35] J. Mei, M. Jiang, Z. Wu, and X. Wang, "Periodically intermittent controlling for finite-time synchronization of complex dynamical networks," Nonlinear Dynamics, vol. 79, no. 1, pp. 295-305, 2014.

[36] F. Yang, J. Mei, and Z. Wu, "Finite-time synchronisation of neural networks with discrete and distributed delays via periodically intermittent memory feedback control," IET Control Theory \& Applications, vol. 10, no. 14, pp. 1630-1640, 2016.

[37] X. Liu, P. Li, and T. Chen, "Cluster synchronization for delayed complex networks via periodically intermittent pinning control," Neurocomputing, vol. 162, pp. 191-200, 2015.

[38] S. Cai, J. Hao, Q. He, and Z. Liu, "Exponential synchronization of complex delayed dynamical networks via pinning periodically intermittent control," Physics Letters A, vol. 375, no. 19, pp. 1965-1971, 2011.

[39] I. Podlubny, "Fractional derivatives and integrals," in Fractional Differential Equations, Academic Press, NY, pp. 41-117, 1999.

[40] N. Aguila-Camacho, M. A. Duarte-Mermoud, and J. A. Gallegos, "Lyapunov functions for fractional order systems," Communications in Nonlinear Science and Numerical Simulation, vol. 19, no. 9, pp. 2951-2957, 2014.

[41] A. A. Kilbas, M. Saigo, and R. K. Saxena, "Generalized mittagleffler function and generalized fractional calculus operators," Integral Transforms and Special Functions, vol. 15, no. 1, pp. 31-49, 2004.

[42] J. Wang and M. FečKan, Y. Zhou, "Presentation of solutions of impulsive fractional langevin equations and existence results," The European Physical Journal Specal Topics, vol. 222, pp. 1857-1874, 2013.

[43] H. G. Wu, S. Y. Chen, and B. C. Bao, "Impulsive synchronization and initial value effect for a memristor-based chaotic system," Acta Physica Sinica, vol. 64, p. 030501, 2015. 


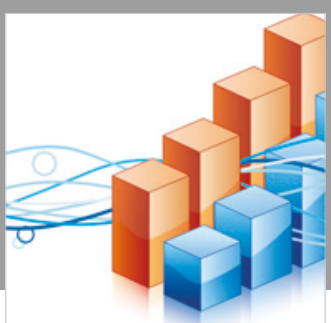

Advances in

Operations Research

\section{-n-m}
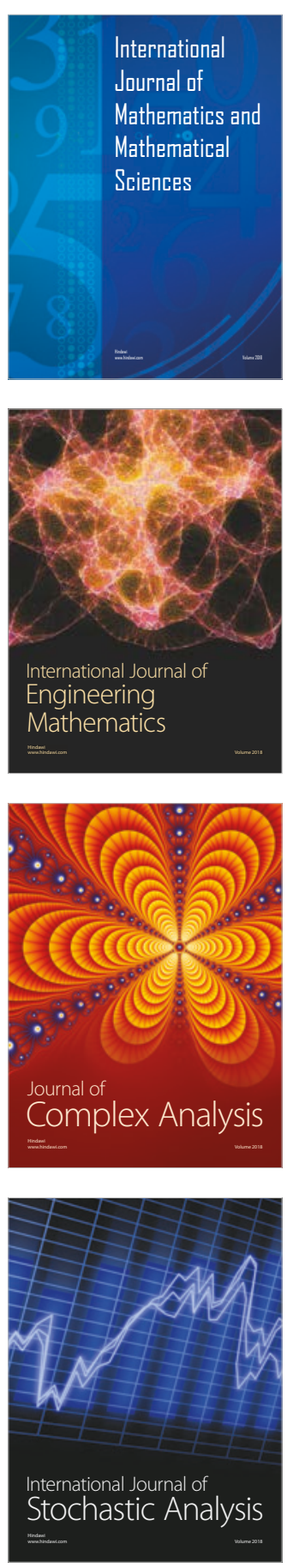
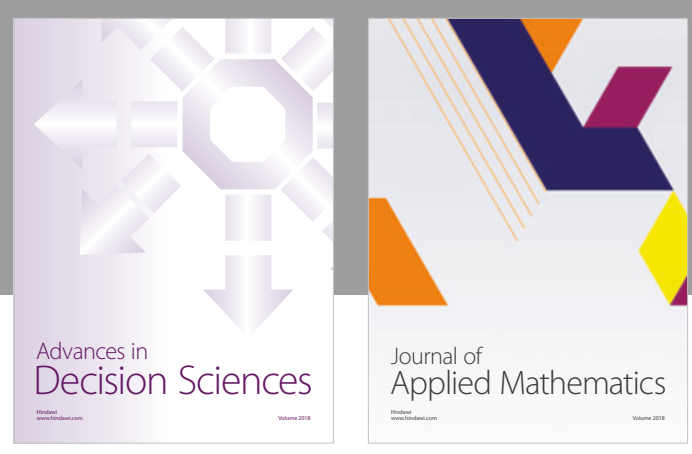

Journal of

Applied Mathematics
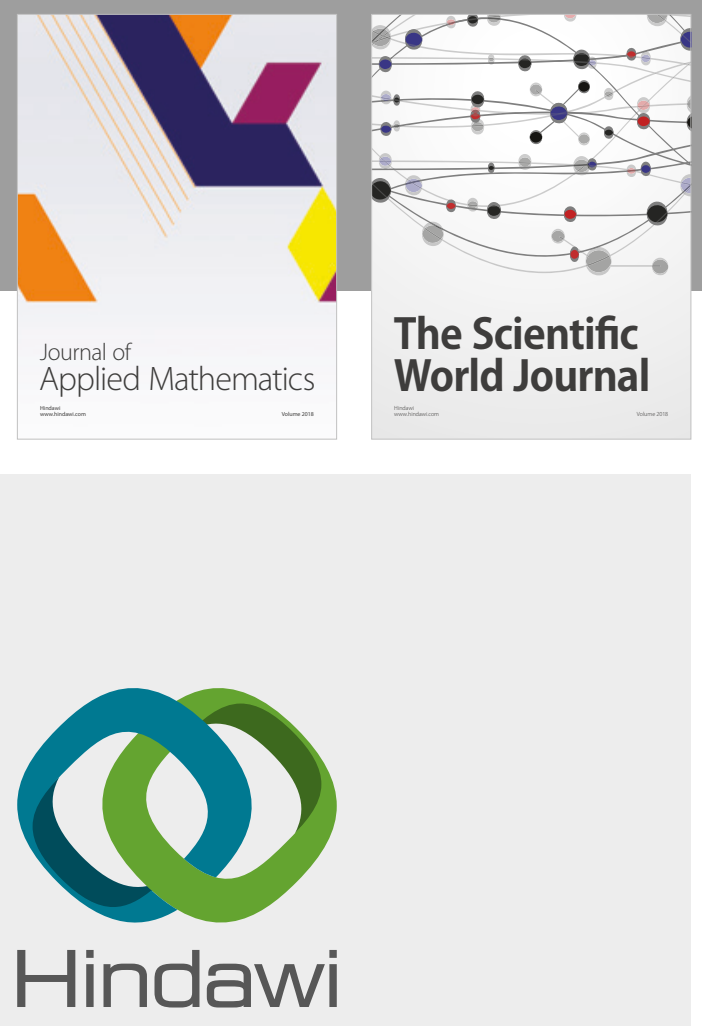

Submit your manuscripts at

www.hindawi.com

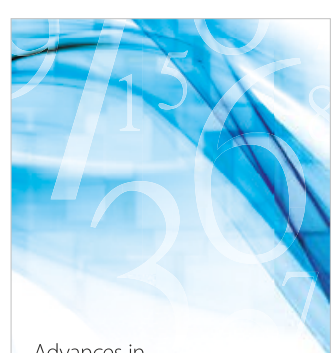

Advances in
Numerical Analysis
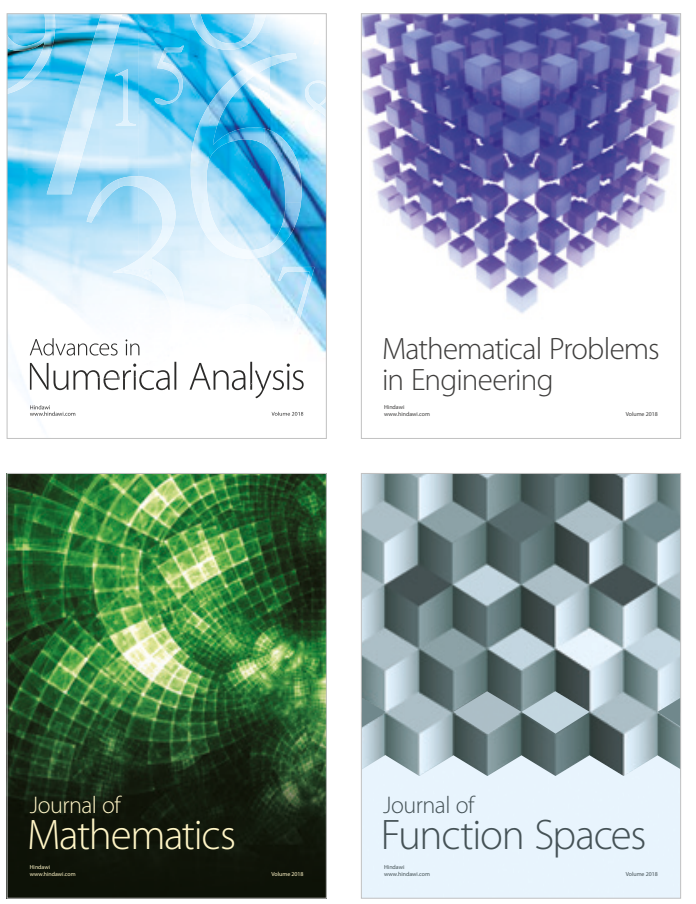

Mathematical Problems in Engineering

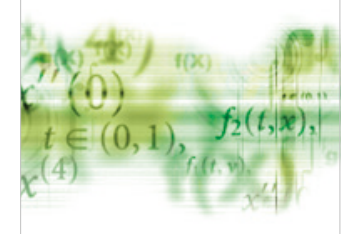

International Journal of

Differential Equations

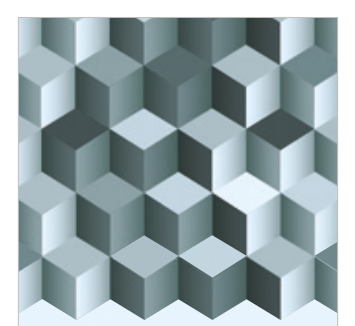

Journal of

Function Spaces
The Scientific

World Journal

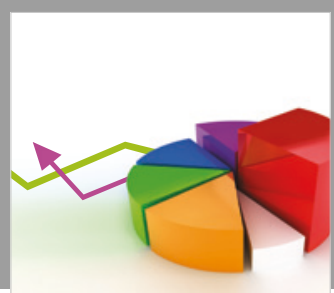

Journal of

Probability and Statistics
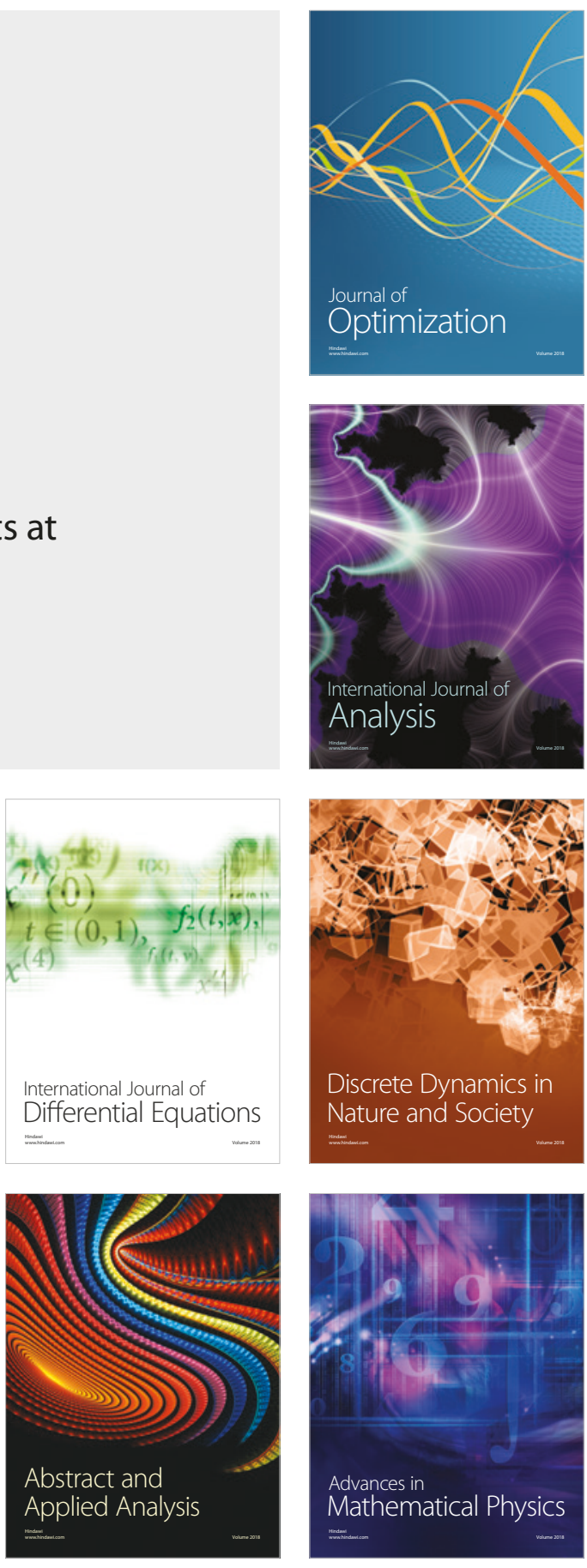